\title{
Can malaria protein fight cancer?
}

\author{
Nevein Naim Fadl, Fathia Abd Elwahid Mannaa*
}

Medical Physiology Department, National Research Centre, Dokki, Cairo, Egypt

*Corresponding author, e-mail: fathia_98@yahoo.com

\begin{abstract}
Cancer risk depends on a combination of our genes, environment and other aspects of our lives, many of which we can control. Surgery, radiation and chemotherapy are often effective at treating people with a solid tumor, but once the cancer has spread and formed tiny tumors at distant sites, chances for a successful recovery are dismal.Recently, it was discovered that the malaria parasite, Plasmodium falciparum produce and present a malarial protein called VAR2CSA, which bind a type of sugar molecule exclusively found in the placenta. It was found that the exact same sugar molecule structure is also found in most cancer cells. Both molecules are a type of chondroitin sulfate. The VAR2CSA only adhere in the placenta and do not bind to chondroitin sulfate expressed elsewhere in the body. In tumors, placental-like chondroitin sulfate chains are linked to a limited repertoire of cancer-associated proteoglycans including CD44 and CSPG4. The recombinant VAR2CSA (rVAR2) protein localizes to tumors in vivo and rVAR2 fused to diphtheria toxin or conjugated to hemiasterlin compounds strongly inhibits in vivo tumor cell growth and metastasis.
\end{abstract}

Key words: Plasmodium falciparum, cancer, malarial protein

\section{Introduction}

Cancer remains a major public health problem, it has a complex pathophysiology. Cancer is an effective family of more than 100 unique conditions and is collectively characterized by the presence of cell populations that undergo uncontrolled division that displays the potential to invade other tissues. In 2014, more than 13 million new cancer cases worldwide were recorded; $70 \%$ of all cancer deaths occurred in low-tomiddle income countries (Soper \& Rasooly, 2016).

Cancer will not be recovered until it is understood and knowing the unique alterations that identify normal cells from tumor cells (Berger et al., 2015). The optimum ideal strategy has not yet been announced. Each one has its own advantages and disadvantages even though a number of them have found their way for clinical application. The combination of strategies can be used to improve the precision of drug delivery, leading to a more effective personalized therapy (Bazak et al., 2015).

Greaves (2014) has a frustrated opinion which may reflect the reality, he believes that most cancers cannot be recuperated and scientists must look for other ways to treat the disease, as developing more advanced cures would lead to cancer cells becoming more resistant to treatment. He added that scientists should focus on prevention, such as aspirin which 
stop the onset of stomach cancer, and respite the disease once it has emerged.

The deadly disease offers the means to another cure, and from this point, a team of researchers accidentally discovered a new protein called malaria protein, Variant Surface antigen 2-CSA (VAR2CSA), produced by the mosquito-borne parasite. VAR2CSA binds to a particular type of sugar molecule present in the placenta, which is also found on the majority of cancer cell types. It was found that, by attaching toxins to VAR2CSA, over 90 per cent of tumor cell lines were effectively cured across a broad array of cancer types such as brain, blood, gastric, lung, skin, prostate, ovarian, pancreatic, bladder, and breast cancers (Salanti et al., 2015).

This article shed light on the role of malaria protein in fighting cancer cells as per the latest research finding.

\section{Cancer}

Cancer cells have a unique physiology.

Human body is made up of more than a hundred million million $(100,000,000,000,000)$ cells. In usual, human body has just the right number of each type of cell. Normal cells produce signals to control how much and how often the cells divide. If any of these signals are faulty or missing, cells may start to grow and multiply too much and form a lump called a tumor (Figure 1). Where the cancer starts is called the primary tumor (Murekatete, 2015). When the cancer cells leave a primary tumor and form one or several secondary tumors in other parts of the body; these secondary tumors are called metastatic tumors or metastases (Vanharanta \& Massague, 2013). Cancer occurs as a result of accumulating genetic and epigenetic alterations in selfrenewing cells that lead to unrestrained cell proliferation, resistance to apoptosis, immune evasion and tumor spread (He \& Sharpless, 2016).

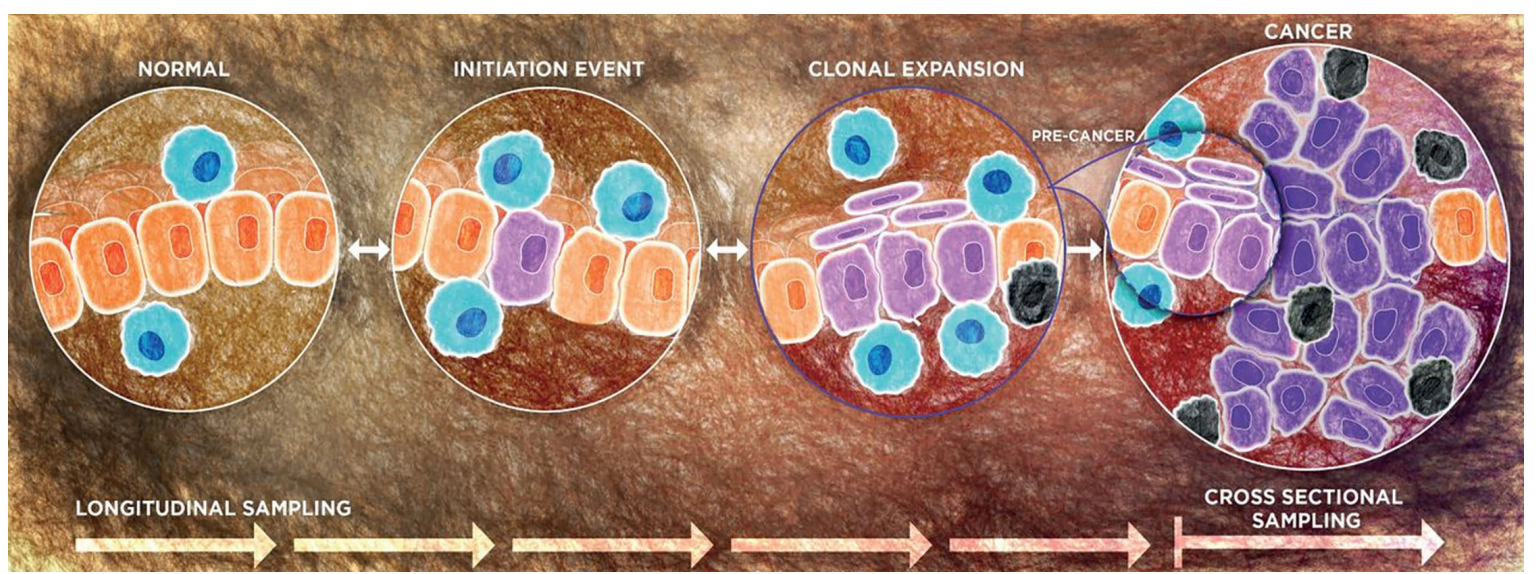

Figure 1. Stages of cancer cell formation. Normal cells (orange), often after long-term exposure to carcinogens, can obtain changes in key molecular pathways and cause uncontrolled proliferation (light purple) leading to a premalignant or precancerous lesion. After accumulating additional alterations, cells are able to invade surrounding tissues forming a tumor (dark purple), in some cases, precancerous lesions naturally regress as marked by the bidirectional arrows. Functional immune cells (blue) with the capability of recognizing and destroying abnormal cells may eventually be repressed (black) by activation of immune checkpoints (Campbell et al., 2016).

Some tissues give rise to cancer in humans a million times more frequently than others. Tomasetti \& Vogelstein (2015) conclude that these differences can be explained by the number of stem cell divisions. They found a strong correlation extending over five orders of magnitude when they plotted the incidence of various cancers against the estimated number of normal stem cell divisions in the corresponding tissues over a lifetime. Tomasetti \& Vogelstein (2015) suggested that random errors occurring during DNA replication in normal stem cells are a major contributing factor in cancer development. Remarkably, this "bad luck" component explains a far greater number of cancers than do hereditary and environmental. This notion was supported by Weinberg \& Zaykin (2015) who mentioned that the importance of errors in DNA replication, has been viewed as implying that most cancers arise through bad luck, leading to the suggestion that research efforts should focus on early detection, rather than etiology or prevention factors (Figure 2). It was found that the major types and most abundant cancers in 
a given population will originate from tissues that have relatively high stem cell division rates and that have a high prevalence of strong relevant risk factors (Giovannucci, 2016).

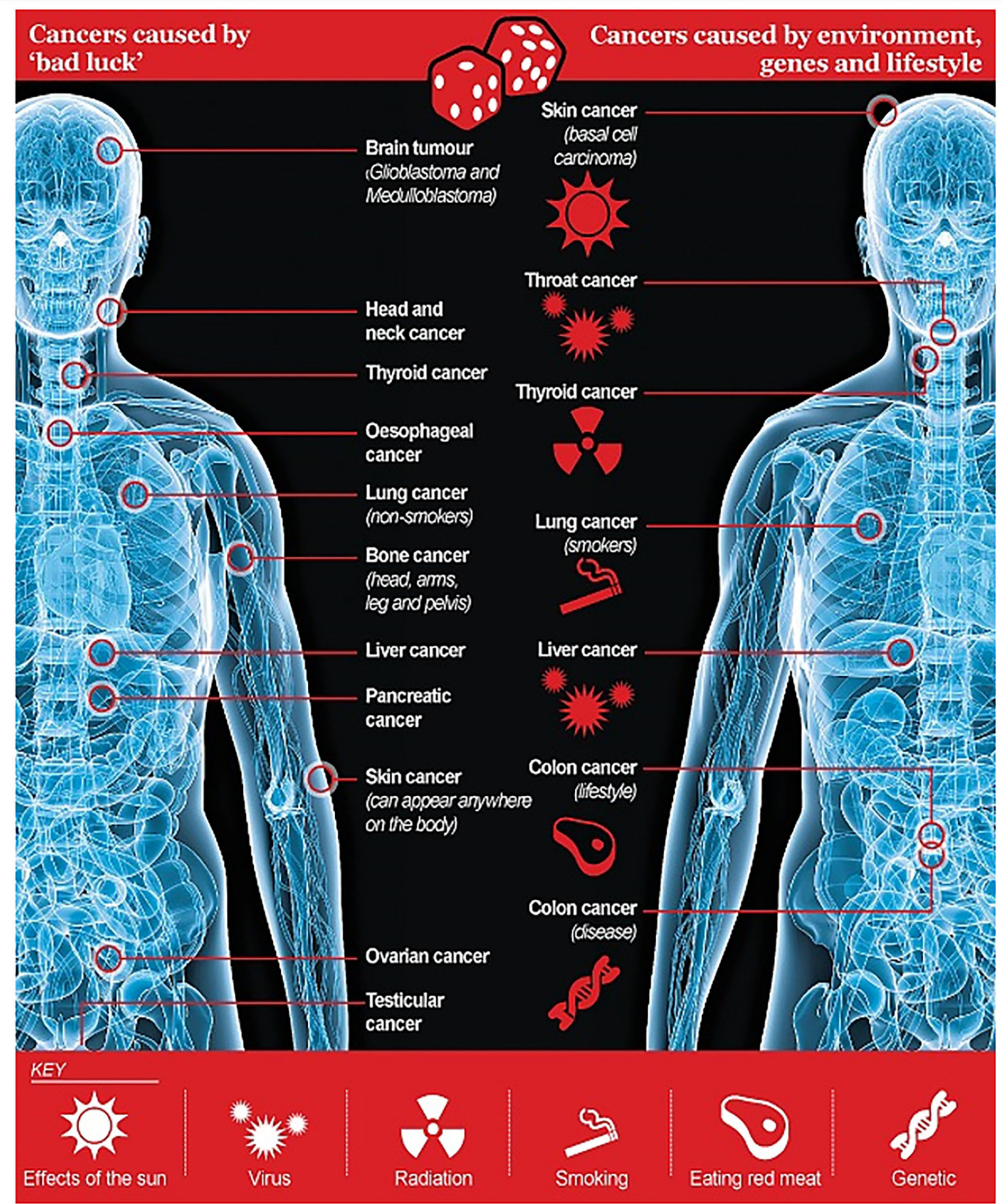

Figure 2. Cancers caused by bad luck and cancer caused by environment, genes and life style. [http://www.telegraph.co.uk/ news/science/science-news/11320497/ (2015)]

The majority of cancer survivors have been treated with aggressive medical, radiation and surgical therapies administered either at a time when a patient's organs were still developing, leading to complications later in life, or when the patient was already suffering from underlying degenerative processes where the side effects of therapy represent harm over injury (Valdivieso et al., 2012). Since1990s, oncologists focused on eliminating all fast-replicating cells, normal or cancerous, by radiation or chemotherapy (Berger el al., 2015).

It was reported that the major challenges in cancer treatment is development 
of agents that can be used for early diagnosis and effective therapy. Classical cancer management frequently lacks accurate tools for early tumors detection and has an associated risk of serious adverse effects of chemotherapeutics. Therefore, the need to optimize therapeutic ratio and have a treatment that recognize and affect cancer cells only (Bazak et al., 2015).

\section{Malaria}

Malaria is one of the world's leading causes of morbidity and mortality with an estimated 438000 malaria deaths and 214 million new cases of malaria worldwide. Malaria is caused by Plasmodium parasites. The parasites are spread to people through the bites of infected female Anopheles mosquitoes, called "malaria vectors". The mosquito bite introduces the parasites from the mosquito's saliva into a person's blood (World Health Organization, 2015).
After that, the parasites migrate to the liver and infect the hepatocytes. Over 7-10 days, the parasites develop and divide into merozoites that are released into the blood stream. During the blood-stage of infection with Plasmodium, the merozoite invades red blood cells (RBCs; reticulocytes and mature erythrocytes) and replicates inside them (Beeson et al., 2016) (Figure3).

According to World Health Organization (2015), there are five parasite species that cause malaria in humans, and two of which Plasmodium falciparum (P. falciparum) and Plasmodium vivax ( $P$. vivax) - confuse the greatest threat. $P$. falciparum is the most prevalent malaria parasite on the African continent. It is responsible for most malaria-related deaths globally, and P. vivax has a wider distribution than $P$. falciparum, and predominates in many countries outside of Africa.

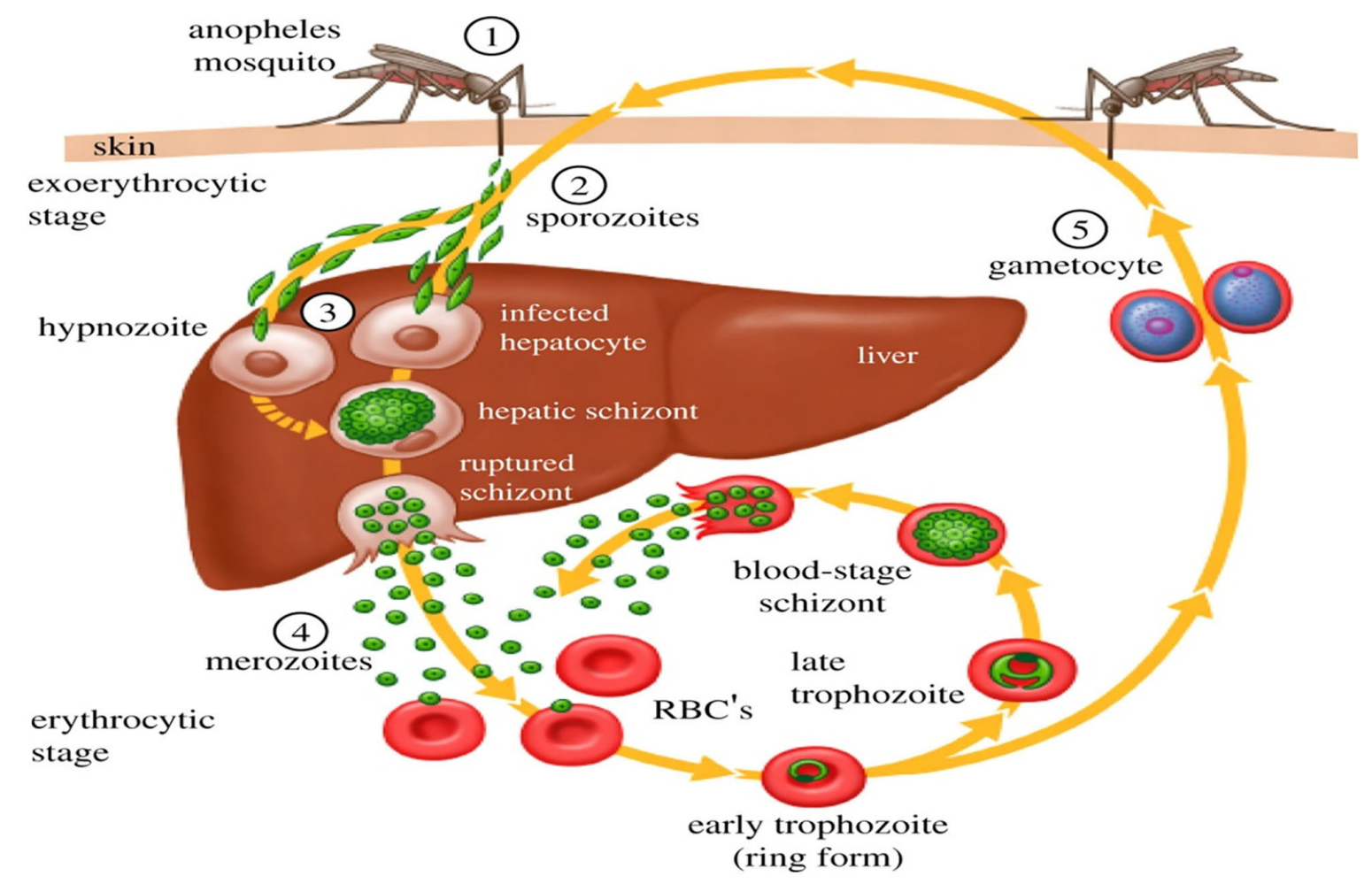

Figure 3. Life cycle of the malaria parasite. [http://rstb.royalsocietypublishing.org/content/366/1579/2806 (2011)]

Malaria was found to affect young children and pregnant women. During pregnancy, malaria is associated with placental infection, maternal anemia, low birth weight infants, and increased neonatal morbidity and mortality (Gnidehou et al., 2014).

For most of its human life cycle, the parasite stays within the liver and blood cells and is relatively invisible to immune surveillance. It avoid destroyed in the spleen by adhesive proteins on the surface of the infected blood cells, causing the blood cells to stick to the walls of small blood vessels, thereby insulate the parasite from passage through the general circulation and 
the spleen (Tilley et al., 2011). The blockage of the microvasculature causes symptoms such as in placental malaria (Mens et al., 2010) and when the sequestered red blood cells breach the blood-brain barrier cause cerebral malaria (Rénia et al., 2012).

\section{Plasmodium falciparum}

Plasmodium falciparum causes the most virulent form of malaria (Liet al., 2006; WHO, 2015). When the parasites invade erythrocyte, several modifications are induced in the infected erythrocyte, especially on its surface membrane. One of the important modifications is the formation of nanoscale protrusions, which are known as knobs (Shi et al., 2013). Knobs are 50 to $120 \mathrm{~nm}$ in diameter and 2 to $20 \mathrm{~nm}$ in height (Quadt et al., 2012) and act as a site for anchoring $P$. falciparum erythrocyte membrane protein 1 (PfEMP1) (Smith et al., 2013) (Figure 4). PfEMPl is a highly polymorphic family of proteins with about 60 members, encoded by the var gene ensemble, that are transported to the surface of parasitized erythrocytes, with their extracellular region exposed for interaction with diverse host receptors (Bentley \& Gamain, 2008).

The role of PfEMP1 is to enable adhesion of infected erythrocyte to various host receptors to avoid splenic clearance, and clonal antigenic variation allows infected erythrocyte to evade immune recognition. For this reason Plasmodium falciparum malaria is more severe than disease caused by other malaria species (Subramani et al., 2015). In this concern, Salanti and his team (2015) observed that $P$. falciparuminfected erythrocytes cannot bind anywhere in vascularized tissue compartments, except in the placenta.

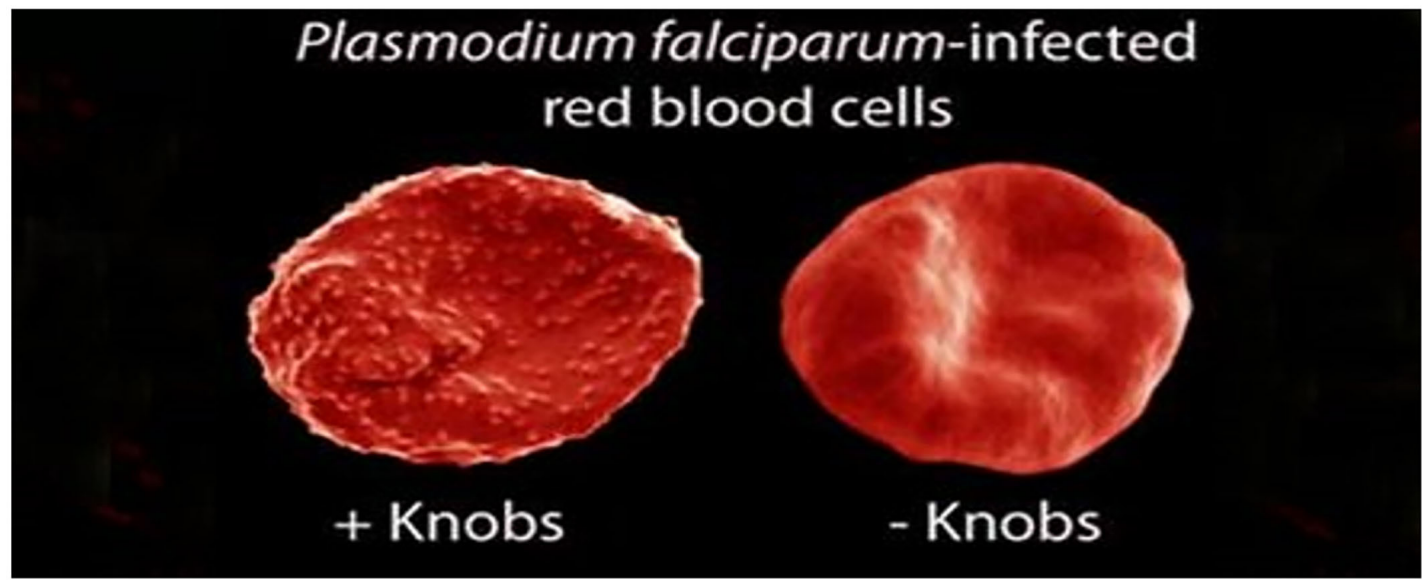

Figure 4. Plasmodium falciparum infected red blood cells. [http://biology.anu.edu.au/research/projects/erythrocytemembrane-modifications-during-malaria-infection (2015)]

VAR2CSA

VAR2CSA, a member of the PfEMP1 variant surface antigen family. VAR2CSA is a large protein $(350 \mathrm{kDa})$ that is structurally composed of six Duffy binding-like (DBL) domains (DBL1X, $D B L 2 X, D B L 3 X, D B L 4 \varepsilon, D B L 5 \varepsilon$, and $D B L 6 \varepsilon$ ) among them, three mediate binding to chondroitin sulfate A (CSA) (Clausen et al., 2012) (Figure 5).

$$
\text { VAR2CSA has extracellular, }
$$

transmembrane, and intracytoplasmic regions, and its extracellular region is uniquely structured among PfEMP1 family members. The extracellular region of VAR2CSA which includes an $\mathrm{N}$-terminal sequence, 6 cysteine-rich Duffy binding like (DBL) domains, and inter-domain (ID) regions, increasingly appear to play a key role in adhesion and immunogenicity of recombinant VAR2CSA protein fragments (Fried \& Duffy, 2015) (Figure 5). PfEMP1 proteins including VAR2CSA are encoded in the genome of $P$. falciparum but not that of other human malaria parasites (Fried \& Duffy, 2015). VAR2CSA expressing parasites only adhere in the placental chondroitin sulfate (CS) and do not bind to CS expressed elsewhere in the body (Salanti et al., 2004; 2015).

\section{Chondroitin Sulfate (CS)}

Chondroitin sulfate (CS) is a glycosaminoglycan (GAG) composed of repeating disaccharides of glucuronic acid and $\mathrm{N}$-acetyl-galactosamine, which are variously substituted by sulfate groups. It is covalently 


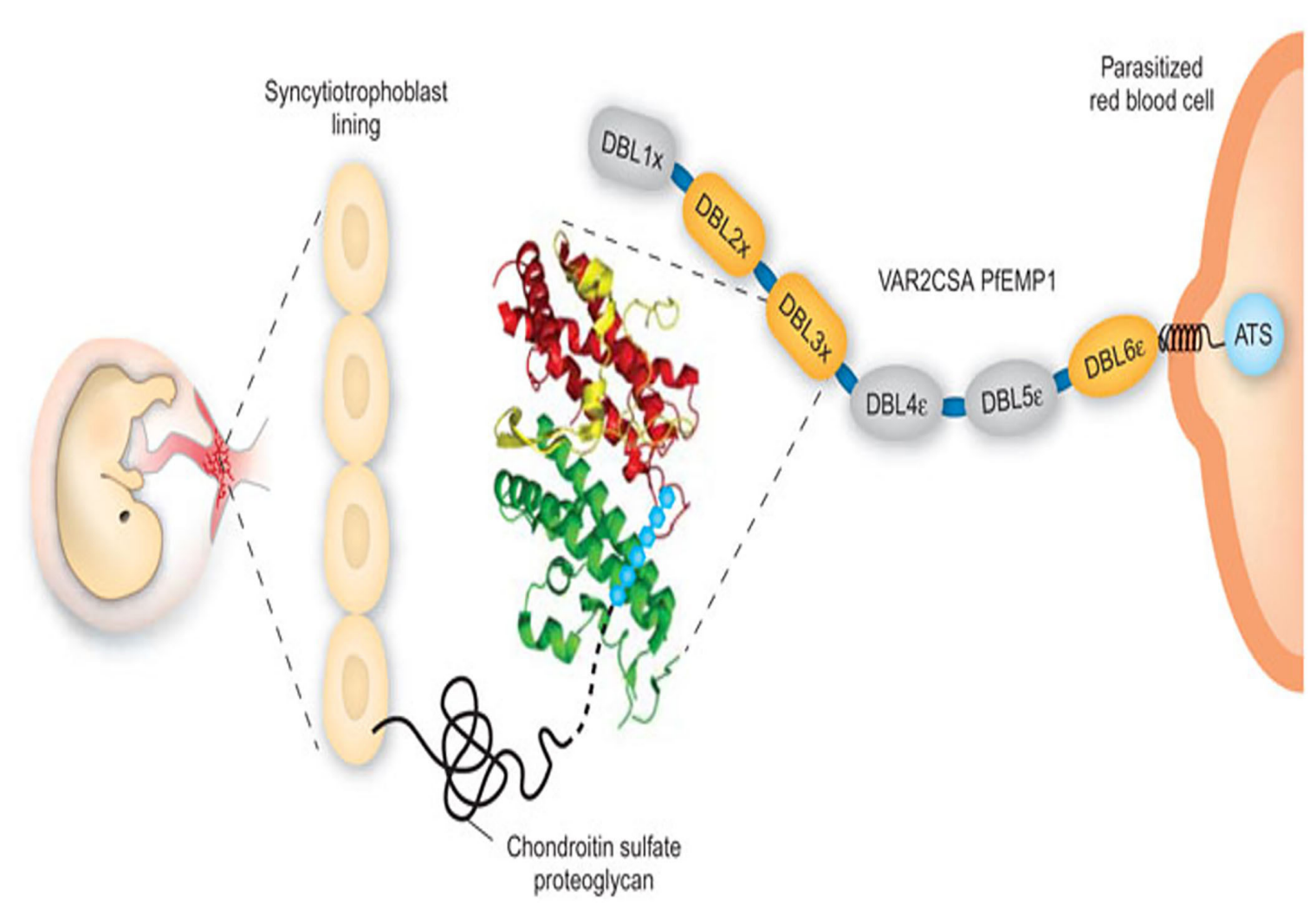

Figure 5. A schematic representation of the VAR2CSA PfEMP1 variant anchored to the membrane of the infected erythrocyte. The DBL3x domain, illustrated as an enlargement in ribbon form. The subdomains 1, 2 and 3 are colored yellow, red and green, respectively. VAR2CSA binds to the CSA moiety of placental chondroitin sulfate proteoglycan present on the surface of the syncytiotrophoblast cells in the placenta. The CSA ligand is shown schematically as hexagons (blue), positioned over the region covering subdomains $2 \& 3$ (Bentley \& Gamain, 2008).

attached on several core proteins creating a variety of proteoglycans (PGs) found in the extracellular matrix (ECM) and cell membrane, but also intracellularly (Elgawish et al., 2015). Chondroitin sulfate interacts with a wide variety of key molecules, such as growth factors, cytokines, chemokines, adhesion molecules and lipoproteins via specific saccharide domains within the chain. These interactions regulate many important physiological processes and cell behavior. Several diseases are often associated with a biosynthetic imbalance of chondroitin sulfate proteoglycans (CSPGs). CSPGs are markedly increased in early atherosclerotic lesions, playing important roles in lipid retention, modification, and finally accumulation. In addition, CSPGs participate in inflammatory process associated with atherosclerosis and influence arterial smooth muscle cell behavior. They also directly affect elastogenesis and proper formation of the extracellular matrix (Theocharis et al., 2006; Poh et al., 2015).

In Pregnancy-associated malaria, an extensive accumulation of parasitized erythrocytes in the placental intervillous blood spaces mediated by adhesion to the placental chondroitin-4-sulfate (CSA) and has been linked to the severe disease outcome (Bentley \& Gamain, 2008).

Plasmodium falciparum-infected red blood cells (IRBCS) bound to chondroitin sulfate A (CSA) (Figure 6) but not to other extracellular matrix proteins or to other known IRBC receptors. IRBCs from non-pregnant donors did not bind to CSA (Fried and Duffy, 1996). This in parallel with Salanti et al (2003) who reported that CSAadhering parasites are rarely found in nonpregnant hosts, placental parasites preferentially or perhaps even exclusively bind to CSA.

The placental CS, although incompletely resolved, is a distinct CS subtype expressed exclusively in the placenta. The function of placental-like CS (pl-CS) chains is not fully understood, but they are associated with the ability of trophoblasts to invade the uterine tissue and promote rapid cell proliferation as part of the 
normal placental implantation process (BastonBust et al., 2010). As proliferation and invasion are features shared with tumor cells, Salanti et al (2015) hypothesized that placenta and cancer might express a similar type of CS.

Notably that the chondroitin sulfate proteoglycans (CSPGs) such as versican accumulate in tumor stroma and play a key role in tumor growth and invasion. The high expression of CSPGs in fast growing tissues and cells is correlated with chondroitin sulfate (CS) chains and the sulfation pattern (Asimakopoulou et al., 2008). The abundance of CSA increased with the advanced stage of the tumor (Ferrarelli, 2015).

Poh et al (2015) mentioned in his article that GAGs have gained interest as potential therapeutic agents in cancer treatment, with studies showing their involvement in various pathobiological cancer stages. An over expression of chondroitin sulfate (CS) has been identified in various cancer phenotypes such as prostate, testicular, gastric, pancreatic and breast cancer. For instance, compositional analysis of GAG side chains isolated from malignant breast tissues indicate an elevation in CS expression, with an increase in CS-A and CS-E sulfation sequences and a decrease in CS-C and CS-D. These indicate that the sulfate groups present on CS might play an important role in the cellular processes involved in the progression of breast cancer. This notion was supported by Guo et al (2015) who mentioned that the percentages of 4-sulfated chondroitin sulfate disaccharide and hyaluronic acid disaccharide in the sera of patients with cirrhosis and hepatocellular carcinoma were significantly increased, compared with healthy individuals. Similarly, Oliveira-Ferrer et al (2015) added that the glycosaminoglycans are abundantly present in ovarian tumors, and there is rising evidence that chondroitin sulfate (CS) as well as diverse carbohydrate sulfotransferases (CHSTs), the enzymes involved in the sulfation process of these structures, plays an important role in metastatic spread of tumor cells.

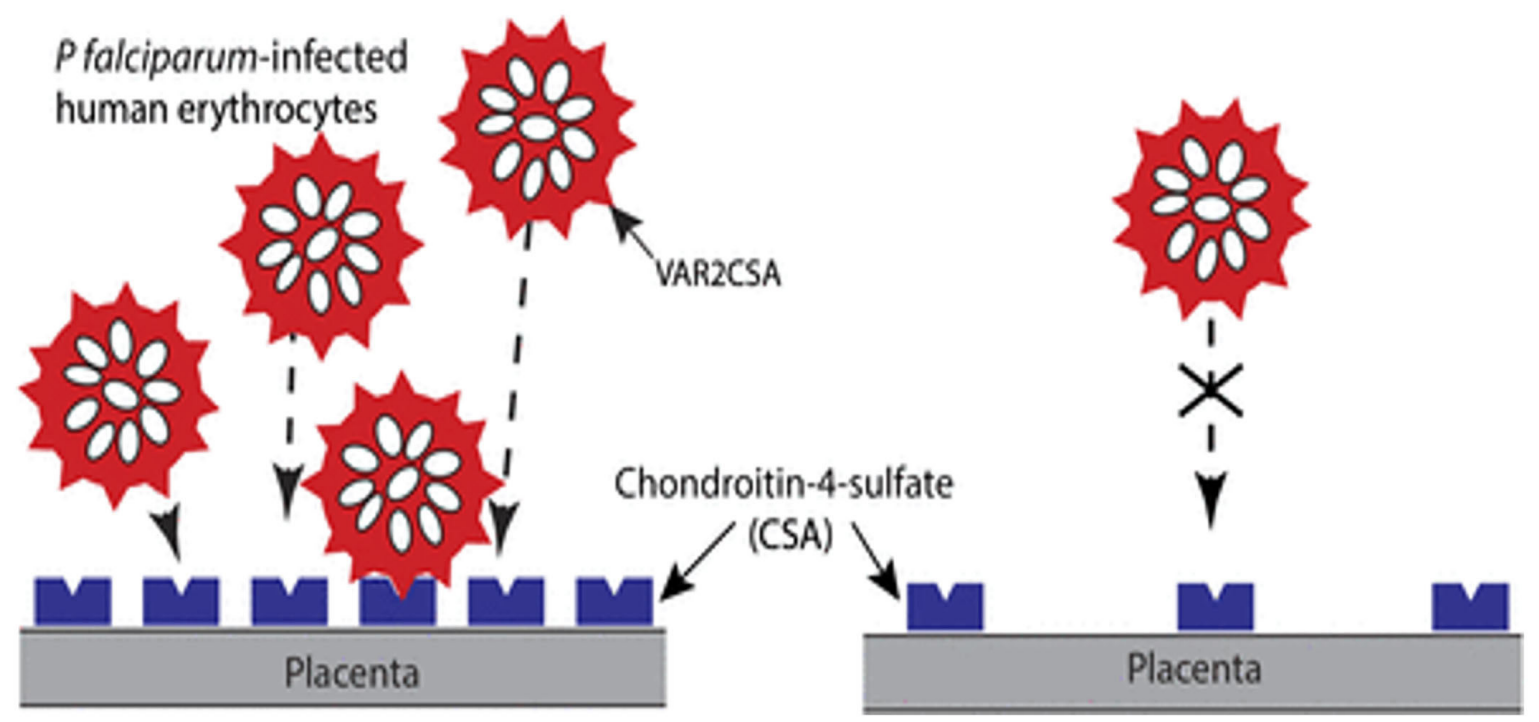

Figure 6. Schematic representation of the to blood capillaries in the human placenta. A variant of PfEMPl encoded by the var2CSA gene, expressed on the surface of infected erythrocytes, binds to CSA, a component of the proteoglycan matrix on placenta (Chishti, 2015).

\section{Pregnancy associated malaria (PAM)}

Pregnancy-associated malaria (PAM) has severe consequences for both mother and child. PAM is caused by $P$. falciparum-infected red blood cells that bind to receptors in the placenta. By adhesion to the placental tissue (sequestration), the parasites avoid being filtered though the spleen where they would have been killed (Andersen et al., 2008). This causes accumulation of infected red blood cells, leading to placental inflammation and block of blood flow to the developing fetus. This is associated with maternal anemia, low birth weight, and premature delivery and can lead to the death of mother and child (Higgins. 2008).

The IEs are however not passed from 
the mother to the fetus, even in cases of heavy infection in which more than $50 \%$ of the erythrocytes are parasitized. They accumulate on the maternal side of the placenta, in the vascular spaces between the villi where nutrient and gas exchange occurs (Wahlgren \& Spillmann., 2000).

The mechanism by which P. falciparuminfected erythrocytes (IEs) sequester in the placenta is mediated by an interactions between $P$. falciparum erythrocyte membrane protein 1 (PfEMP1) domains (variant protein named VAR2CSA) and chondroitin sulfate A (CSA) on the syncytiotrophoblasts (Figure 6) of placenta (Andersen et al., 2008; Gnidehou et al., 2014).

Is there a relation between infection with malaria and incidence of cancer?

In 2010, Lehrer reported that there was a significant association between malaria and cancer mortality in 50 US states. The association can be possibly explained by the well established ability of Plasmodium to induce suppression of the immune system. A second explanation may be that the Anopheles mosquito, the vector of malaria, transmits an obscure virus that initially causes only a mild transitory illness but much later predisposes to cancer.

On the other hand, Chen et al (2011) reported that Malaria infection significantly suppresses Lewis lung cancer growth in a mouse model by inducing both a potent anti-tumor innate immune response, including the secretion of IFN-Y and TNF- $a$ and the activation of natural killer cells as well as adaptive anti-tumor immunity with increasing tumor-specific T-cell proliferation and cytolytic activity of $\mathrm{T}$ cells. He suggested that the malaria parasite may provide a novel strategy or therapeutic vaccine vector for antilung cancer immune-based therapy.

Recently, Robbiani et al (2015) decided to find out the link between the parasite that causes malaria, infects red blood cells and liver cells, and increase a child's risk of developing of one of the most frequent cancers among African children called Burkitt's lymphoma. Robbiani et al (2015) studied malaria infections in mice and discovered that during prolonged immune responses to malaria, B lymphocytes multiply extensively and show prolonged expression of an enzyme called AID (activation-induced cytidine deaminase). Normally, AID mutates DNA at antibody genes to promote shuffling of DNA, which generates the diversity of antibodies so crucial for combating infections. However, Robbiani's team found that in malaria-infected $B$ lymphocytes; AID instead revenge widespread devastation, causing DNA rearrangements at other genes, including those involved in the development of cancer.

\section{Treatment of cancer with malaria protein}

In the recent decades, scientists have been searching for similarities between the growth of a placenta and a malignant compartment (Holtan et al., 2009). The placenta is a fast-growing organ in which cells display high mitotic rates and within a few months grows from only few cells into an organ weighing approximately two pounds and it provides the embryo with oxygen and nourishment in a relatively foreign environment. Placenta also has the ability to maintain high proliferation rates and the capacity of the villous trophoblasts to invade into uterine tissue during implantation (BastonBust et al., 2010; Van Sinderen et al., 2013), these are features shared with tumors, and they grow aggressively in a relatively foreign environment (Salanti et al., 2015).

Now as per the latest research findings, when researchers (Salanti et al., 2015) were trying to develop an anti-malaria vaccine in pregnant women they discovered an unexpected side benefit that could potentially transform cancer therapy. They revealed that the carbohydrate that the malaria parasite attaches itself to - an oncofetal chondroitin sulfate (CSA) - which is expressed in the placenta is the same as the one displayed on proteoglycans in cancerous cells (the placental and malignant compartments display a common CS signature that binds malarial protein,VAR2CSA,) (Figure 7).

Salanti and his colleagues (2015) suggested that the sugar molecule (CSA) could be a target for anti-cancer drugs, and that the VAR2CSA malarial protein could provide the tool for delivering cancer killing toxins to tumors lattach a poison-carrying protein from the malaria parasite directly onto the cancer cell, 
thereby killing the cancer).

To examine this idea, the researchers (Salanti et al., 2015) separated the malaria protein (VAR2CSA), which attaches itself to the carbohydrate (CSA) and chemically conjugated it to a diphtheria toxin (rVAR2), (Figure 7) then tested in vitro on a thousands of samples from brain tumors to leukemia's, the general picture emerges to indicate that the malaria protein is able to attack more than $90 \%$ of all types of tumors. Researchers took their drug one step further and tested it out on mice that were implanted with three different human cancers. With non-Hodgkin's lymphoma, the treated mice's tumors were about a quarter the size of the tumors in the control group. With prostate cancer, the tumors disappeared in two of the six treated mice a month after receiving the first dose. With metastatic bone cancer, five out of six of the treated mice were alive after almost eight weeks, compared to none of the mice in a control group (Figures 8 e 9).

\section{Malaria}

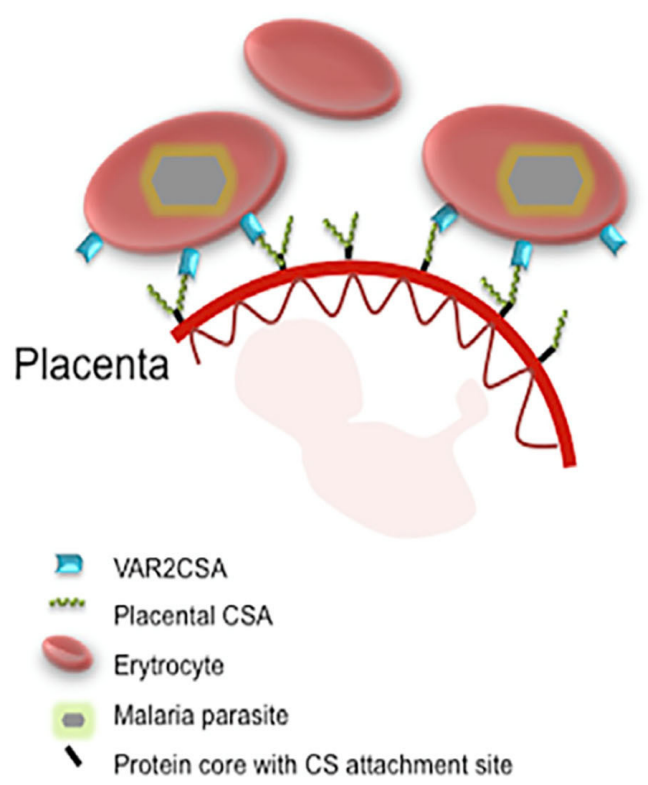

\section{Cancer}

Therapeutic targeting Diagnostic targeting

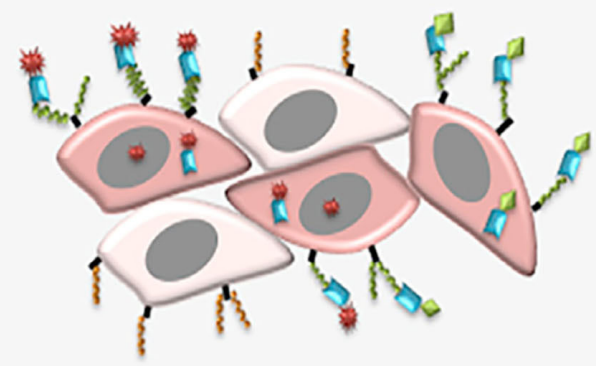

Recombinant VAR2CSA
Oncofetal CSA
Normal CSA
Tumor cell
Normal cell
w Drug/toxin
Wabel

Figure 7. The malarial protein VAR2CSA binds a placenta-specific chondroitin sulfate (CS). Same CS is present in high fractions of cancer cells of many cancer types and that recombinant VAR2CSA conjugated with therapeutics strongly inhibit in vivo tumor growth (Salanti et al., 2015).

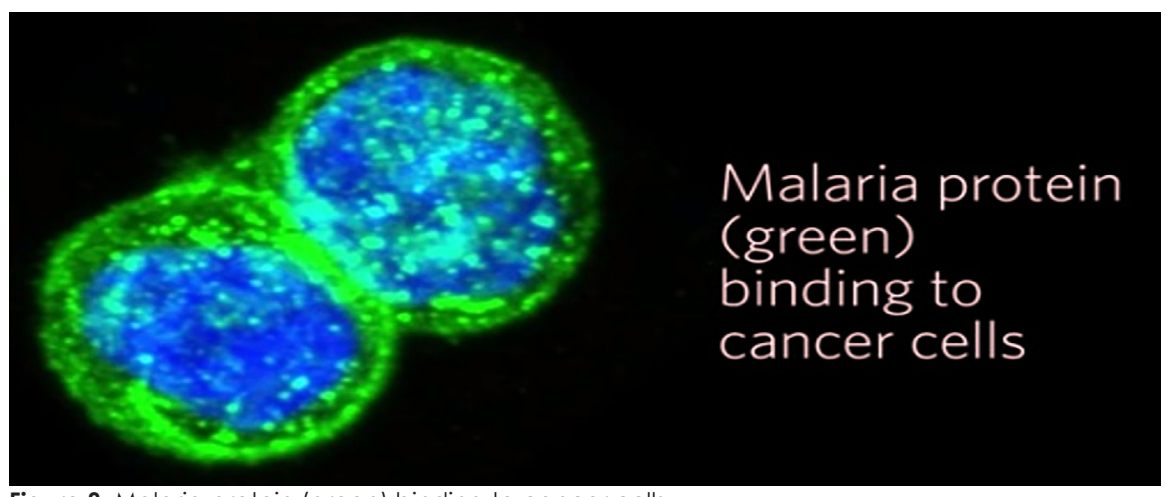

Figure 8. Malaria protein (green) binding to cancer cells.

http://www.zmescience.com/medicine/malaria-cancer-trials-20102015/ (2015) 
Histopathological examination of different organs did not show any evidence of adverse cytotoxic effects. These data demonstrate that rVAR2 can facilitate efficacious CS dependent delivery of a cytotoxic compound to tumors in vivo, rVAR2 were able to inhibit tumor growth and metastasis with no orphologic evidence of adverse effects on normal tissues (Bernstein, 2015; Salanti et al., 2015).

Obviously, this treatment cannot be applied to pregnant women. Since the rVAR2conjugated toxin cannot distinguish placenta from tumor, it would inhibit placenta growth as well (Salanti et al., 2015).

According to the opinion of Salanti and his team (2015), he concluded that his study offered compelling evidence that diverse human and murine tumor types can be effectively targeted in vivo using an rVAR2 drug conjugate, and this is supported by the observation that $P$. falciparum-infected erythrocytes cannot bind anywhere in vascularized tissue compartments, except in the placenta.

On the other hand, Lassen (2015), although he is exciting with mechanism involved in using rVAR2 drug, and the results of animal tests, but he emphasized that the cancer treatment will not necessarily work in human, as, it is easier to treat mice than people and unforeseen side effects may yet emerge when tested on cancer patients.

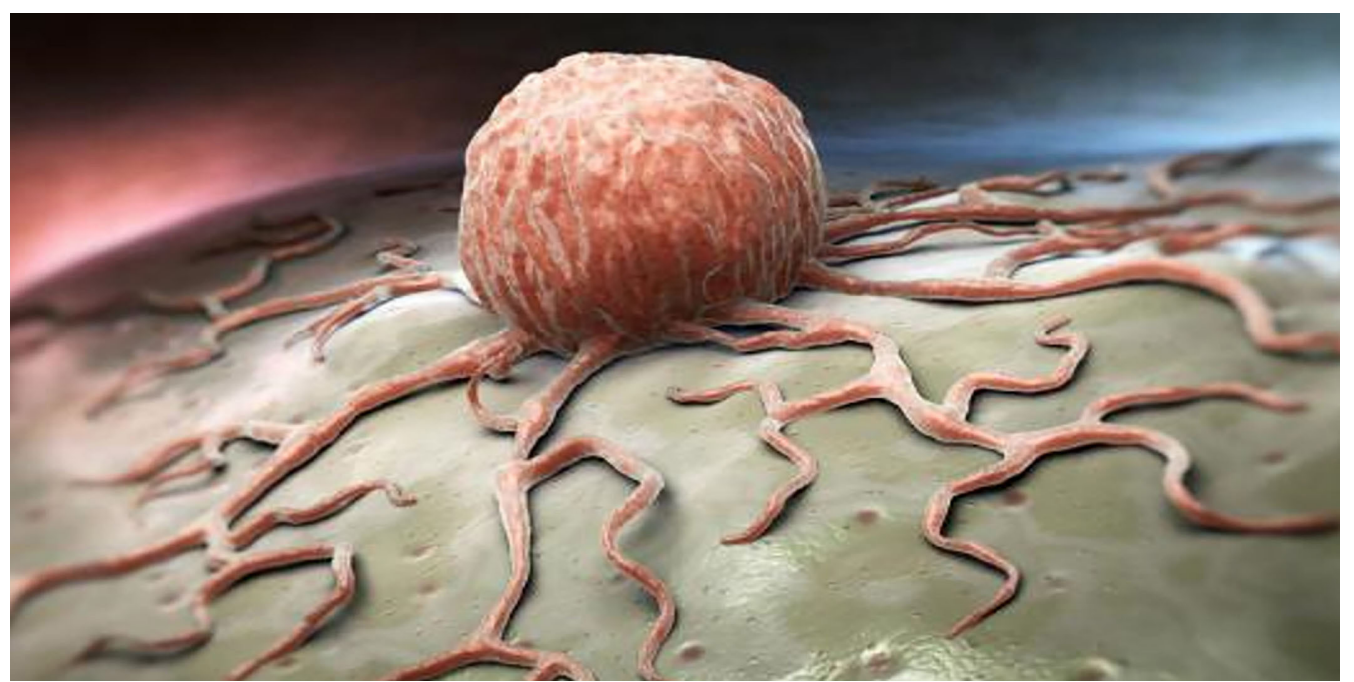

Figure 9. Malaria proteins with toxins attached appeared to target cancer cells, and not healthy ones, during experiments with mice. http://www.upi.com/Health_News/2015/10/14/Malaria-vaccine-research-may-lead-tocure-for-cancer/5851444835216/ (2015)

\section{Conclusion}

Cancer risk factors include exposure to chemicals or other substances, as well as certain behaviors. They also include things people cannot control, like age and family history. A family history of certain cancers can be a sign of a possible inherited cancer syndrome.

Surgery, radiation and chemotherapy are often effective at treating people with a solid tumor but once the cancer has spread (metastases) and formed tiny tumors at distant sites, chances for a successful recovery are dismal and it represented 90 percent of people who die from cancer.

Group of Danish scientists from the University of Copenhagen and the University of British Columbia, while trying to develop an anti-malaria vaccine in pregnant women, they discovered that the malaria parasite, Plasmodium falciparum produce and present a malarial protein called VAR2CSA, which binds a distinct type sugar called chondroitin sulfate (CS) exclusively expressed in the placenta. The same CS modification is present on a high proportion of cancer cells and that it can be specifically targeted by recombinant VAR2CSA (rVAR2). In tumors, placental-like CS chains are linked to a limited repertoire of cancer-associated proteoglycans including CD44 and CSPG4. The rVAR2 protein localizes to tumors in vivo and rVAR2 fused to diphtheria toxin or conjugated to hemiasterlin compounds strongly inhibits in vivo 
tumor cell growth and metastasis.

Out of the idea "take a small component of a fetal disease and then used it to target another disease", By using the same mechanism of binding of the malaria protein (VAR2CSA) to the sugar molecule (CSA), they tested a drug that combines the malaria protein with an anticancer toxin, first in cell lines and then in mice. In cell lines, they found that the combination drug specifically targeted and killed more than $90 \%$ of cancer cell lines. And in mice implanted with three types of human tumors - the drug also showed varying degrees of success. In mice with non-Hodgkin's lymphoma, the treated tumors shrank to a quarter of the size of untreated tumors. Treated mice within a month of administering the first dose, and with metastatic breast cancer, five of six treated mice were cured of the disease. In all groups the mice showed no adverse side effects from the treatment and their organs were unharmed by it.

The current drug of choice to treat cancer is chemotherapy, which kills the healthy cells while trying to kill the cancer cells. With this conjugate of malaria protein and toxin, it possible to produce a treatment capture and kill only the cancer cells in the body.

The biggest questions are, whether it'll work in the human body, and if the human body can tolerate the doses needed without developing side effects?. Further researches in animal models are needed to review the pathophysiological pattern of this conjugate before human trials can commence.

\section{References}

Andersen, P., Nielsen, M.A., Resende, M., Rask, T.S., Dahlbäck, M., Theander, T., Lund, O., Salanti, A. 2008. Structural insight into epitopes in the pregnancy-associated malaria protein VAR2CSA. PLOS Pathog 4(2):e42. doi: 10.1371/ journal.ppat.0040042.

Asimakopoulou, A.P., Theocharis, A.D., Tzanakakis, G.N., Karamanos, N.K . 2008. The biological role of chondroitin sulfate in cancer and chondroitinbased anticancer agents. In Vivo 22(3):385-9.

Baston-Büst, D.M ., Götte, M ., Janni, W., Krüssel, J.S., Hess, A.P. 2010. Syndecan-1 knock-down in decidualized human endometrial stromal cells leads to significant changes in cytokine and angiogenic factor expression patterns. Reprod.
Biol. Endocrinol 8: 133. doi: 10.1186/1477-7827-8133.

Bazak, R., Houri, M., El Achy, S., Kamel, S., Refaat, T. 2015. Cancer active targeting by nanoparticles: a comprehensive review of literature. J Cancer Res Clin Oncol 141(5):769-84.

Beeson, J.G., Drew, D.R., Boyle, M.J., Feng, G., Fowkes, FJI., Richards, J.S. 2016. Merozoite surface proteins in red blood cell invasion, immunity and vaccines against malaria. FEMS Microbiology Reviews. doi: http://dx.doi.org/10.1093/femsre/ fuw001 fuw001.

Bentley, G.A., Gamain, B. 2008. How does Plasmodium falciparum stick to CSA? Let's see in the crystal. Nature Structural \& Molecular Biology 15: 895 - 897.

Berger, T., Saunders, M.E., Mak, T.W. 2015. Fighting Fire with Fire in Cancer. In: Innovative Medicine, Nakao, K. et al (eds). pp 39-49. doi: 10.1007/978-4-431-55651-0 3 .

Bernstein, L.H. 2015. Malaria protein anti-cancer activity. http://www.genscript.com/protein_ news.htm.

Campbell, J.D., Mazzilli, S.A., Reid, M.E., Dhillon, S.S., Platero, S., Beane, J., Spira, A.E. 2016. The case for a pre-cancer genome atlas (PCGA). Cancer Prev Res 9:119-124.

Chen, L., He, Z., Qin, L., Li, Q., Shi, X., Zhao, S., Chen, L., Zhong, N., Chen, X. 2011. Antitumor effect of malaria parasite infection in a murine Lewis lung cancer model through induction of innate and adaptive immunity. PLOS One 6(9):e24407. doi: 10.1371/journal.pone.0024407.

Chishti, A.H. 2015. Malaria selectively targets pregnancy receptors. Blood 125(2): 217-218.

Clausen, T.M., Christoffersen, S., Dahlbäck, M., Langkilde, AE., Jensen, KE., Resende, M., Agerbaek, M.Ø., Andersen, D., Berisha, B., Ditlev, S.B., Pinto, V.V., Nielsen, M.A., Theander, T.G., Larsen, S., Salanti, .2012. Structural and functional insight into how the Plasmodium falciparum VAR2CSA protein mediates binding to chondroitin sulfate A in placental malaria. J. Biol. Chem 287:2333223345.

Elgawish, M.H., Zakaria, M.A., Fahmy, H.S., Shalaby, A.A . 2015. Effect of chondroitin sulfate on cartilage volume loss and subchondral bone marrow lesions in osteoarthritis knee. Egyptian Rheumatology \& Rehabilitation 42(3): 153-158.

Ferrarelli, L.K. 2015. Finding the good in malaria. Science Signaling 8(399): ec297. doi: 10.1126/ scisignal.aad6490.

Fried, M., Duffy, P.E. 2015. Designing a VAR2CSA- 
based vaccine to prevent placental malaria. vaccine 23(2): 7483-7488.

Fried, M., Duffy, P.E.1996. Adherence of Plasmodium falciparum to chondroitin sulphate $A$ in the human placenta. Science 272: 1502-1504.

Giovannucci, E.L. 2016. Are most cancers caused by specific risk factors acting on tissues with high underlying stem cell divisions?. J Natl Cancer Inst 108(3): 10.1093/jnci/djv343.

Gnidehou, S., Doritchamou, J., Arango, E.M., Cabrera, A., Arroyo, M.l., Kain, K.C., Ndam, N.T., Maestre, A., Yanow, S.K. 2014. Functional antibodies against VAR2CSA in nonpregnant populations from colombia exposed to Plasmodium falciparum and Plasmodium vivax. Infect Immun 82(6):2565-73.

Greave, M . 2014. http://www.telegraph. co.uk/news/science/science-news/11062255/ Lets-stop-trying-to-cure-cancer-says-cancerprofessor.html.

Guo, S., Xue, C., Li, G., Zhao, X., Wang, Y., Xu, J. 2015. Serum Levels of glycosaminoglycans and chondroitin sulfate/hyaluronic acid disaccharides as diagnostic markers for liver diseases. Journal of Carbohydrate Chemistry 34(2): 55-69.

He, S. \& Sharpless, N.E. 2016. The impact of aging on cancer progression and treatment. Advances in Geroscience. (book), pp 53-83. (C) Springer International Publishing AG, Part of Springer Science+Business Media.

Higgins, M.K. 2008. The structure of a chondroitin sulfate-binding domain important in placental malaria. J Biol Chem 283(32): 21842-21846.

Holtan, S.G., Creedon, D.J., Haluska, P., Markovic, S.N. 2009. Cancer and pregnancy: parallels in growth, invasion, and immune modulation and implications for cancer therapeutic agents. Mayo Clin. Proc 84: 985-1000.

Lassen, U. 2015. http://sciencenordic.com/ malaria-protein-targets-and-kills-cancer-cells.

Lehrer, S. 2010. Association between malaria incidence and all cancer mortality in fifty U.S. States and the District of Columbia. Anticancer Res 30(4):1371-3.

Li, A., Mansoor, A.H., Tan, K.S., Lim, C.T. 2006. Observations on the internal and surface morphology of malaria infected blood cells using optical and atomic force microscopy. J Microbiol Methods 66:434-439.

Mens, P.F., Bojtor, EC., Schallig, HD. 2010. Molecular interactions in the placenta during malaria infection. European Journal of Obstetrics \& Gynecology and Reproductive Biology 152 (2):
$126-32$.

Murekatete, k. 2015. Raising awareness of lifestyle factors as an important part of a person's risk of breast cancer. World Congress on Breast Cancer, Birmingham, UK. August 03-05, 2015 Birmingham, UK. doi: 10.4172/1948-5956.S1.042.

Oliveira-Ferrer, L., Heßling, A., Trillsch, F., Mahner, S., Milde-Langosch, K. 2015. Prognostic impact of chondroitin-4-sulfotransferase CHST11 in ovarian cancer. Tumor Biology 36(11): 90239030.

Poh, Z.W., Gan, C.H., Lee, E.J., Guo, S., Yip, G.W., Lam, Y. 2015. Divergent synthesis of chondroitin sulfate disaccharides and identification of sulfate motifs that inhibit triple negative breast cancer. Scientific Reports 5, Article number: 14355. doi:10.1038/srep 14355 .

Quadt, K.A., Barfod, L.,Andersen, D., Bruun, J., yan, B., Hassenkam, T., Ofori, M.F., Hviid, L. 2012. The density of knobs on Plasmodium infected erythrocytes depends on developmental age and varies among isolates. PLOS One 7:e45658. 10.1371/journal.pone.0045658.

Rénia, L., Howland, S.W., Claser, C., Gruner, A., Suwanarusk, R., Hui Teo, T., Russell, B., Ng, L.F. 2012. Cerebral malaria: mysteries at the bloodbrain barrier. Virulence 3 (2): 193-201.

Robbiani, D.F., Deroubaix, S., Feldhahn, N., Oliveira, T.Y., Callen, E., Wang, Q., Jankovic, M., Silva, I.T., Rommel, P.C., Bosque, D., Eisenreich, T., Nussenzweig, A., Nussenzweig, M,C. 2015. Plasmodium infection promotes genomic instability and AID-dependent B cell lymphoma. Cell 162(4):727-37.

Salanti, A., Dahlback, M., Turner, L., Nielsen, MA., Barfod, L., Magistrado, P., Jensen, ATR., Lavstsen, T., Ofori ,MF., Marsh, K. , Hviid, L., Theander, TG. 2004. Evidence for the involvement of VAR2CSA in pregnancy-associated malaria. J Exp Med 200: 1197-1203.

Salanti, A., Staalsoe, T., Lavstsen, T., Jensen, A.T., Sowa, M.P., Arnot, D.E., Hviid, L., Theander, T.G. 2003. Selective upregulation of a single distinctly structured var gene in chondroitin sulphate A-adhering Plasmodium falciparum involved in pregnancy-associated malaria. Mol Microbiol 49: 179-191

Salanti, A., Clausen, T.M., Agerbaek, M.Ø., Al Nakouzi, N., Dahlbäck, M., Oo, H.Z., Lee, S., Gustavsson, T., Rich, J.R., Hedberg, B.J., Mao, Y., Barington, L., Pereira, M.A., LoBello, J., Endo, M., Fazli, L., Soden, J., Wang, C.K., Sander, A.F., Dagil, R., Thrane, S., Holst, P.J., Meng, L., Favero, F., Weiss, G.J., Nielsen, M.A., Freeth, J., Nielsen, T.O., Zaia, J., Tran, N.L., Trent, J., Babcook, J.S., Theander, T.G., 
Sorensen, P.H., Daugaard, M. 2015. Targeting human cancer by a glycosaminoglycan binding malaria protein. Cancer Cell 28(4):500-514.

Shi, H., Liu, Z., Li, A., Yin, J., Chong, A.G., Tan, K.S., Zhang, Y., Lim, C.T. 2013. Life cycle-dependent cytoskeletal modifications in Plasmodium falciparum infected erythrocytes. PLOS One 8:e61170. doi: 10.1371/journal.pone.0061170.

Smith, J.D., Rowe, J.A., Higgins, M.K., Lavstsen, T. 2013. Malaria's deadly grip: cytoadhesion of Plasmodium falciparum-infected erythrocytes. Cell Microbiol 15:1976-1983.

Soper, S.A. \& Rasooly, A. 2016. Cancer: a global concern that demands new detection technologies. Analyst 141: 367-370.

Subramani, R., Quadt, K., Jeppesen, AE., Hempel, C., Petersen, JE., Hassenkam, T., Hviid, L., Barfod, L. 2015. Plasmodium falciparuminfected erythrocyte knob density is linked to the PfEMP1 variant expressed. mBio. 6(5):e01456-15. doi: 10.1128/mBio.01456-15 6 October 2015 mBio vol. 6 no. 5 e01456-15.

Theocharis, A.D., Tsolakis, I., Tzanakakis, G.N., Karamanos, N.K. 2006. Chondroitin sulfate as a key molecule in the development of atherosclerosis and cancer progression. Adv Pharmacol 53:28195.

Tilley, L., Dixon, M.W., Kirk, K. 2011 . The Plasmodium falciparum-infected red blood cell. International Journal of Biochemistry and Cell Biology 43 (6): 839-42.

Tomasetti, C., Vogelstein, B. 2015. Variation in cancer risk among tissues can be explained by the number of stem cell divisions. Science 347:78-81.

Valdivieso, M., Kujawa, A.M., Jones, T., Baker, L.H . 2012. Cancer survivors in the united states: $A$ Review of the Literature and a Call to Action. Int J Med Sci 9(2):163-173.

Van Sinderen, M., Cuman, C., Winship, A., Menkhorst, E., Dimitriadis, E. 2013. The chrondroitin sulfate proteoglycan (CSPG4) regulates human trophoblast function. Placenta 34: 907-912.

Vanharanta, S., Massague, J. 2013. Origins of metastatic traits. Cancer Cell 24(4):410-421.

Wahlgren, M., Spillmann, D. 2000. Sticky sugars attract malaria to the fetus. Nature Medicine $6: 25-26$.

Weinberg, C.R., Zaykin, D. 2015. Is bad luck the main cause of cancer?. J Natl Cancer Inst ., 107(7): djv125.doi: 10.1093/jnci/djv125.

World Health Organization. 2015. WHO Fact
Sheet: World Malaria Report, 9 December 2015. http://www.who.int/malaria/publications/worldmalaria-report-2015/report/en/ 